\title{
Estudio de la formabilidad en caliente de aleaciones de titanio a través del ensayo de torsión y su aplicación a la optimización de procesos de forja y extrusión
}

\author{
Study of hot-workability of titanium alloys through \\ hot-torsion testing and its application to the \\ optimization of forging and extrusion \\ processes
}

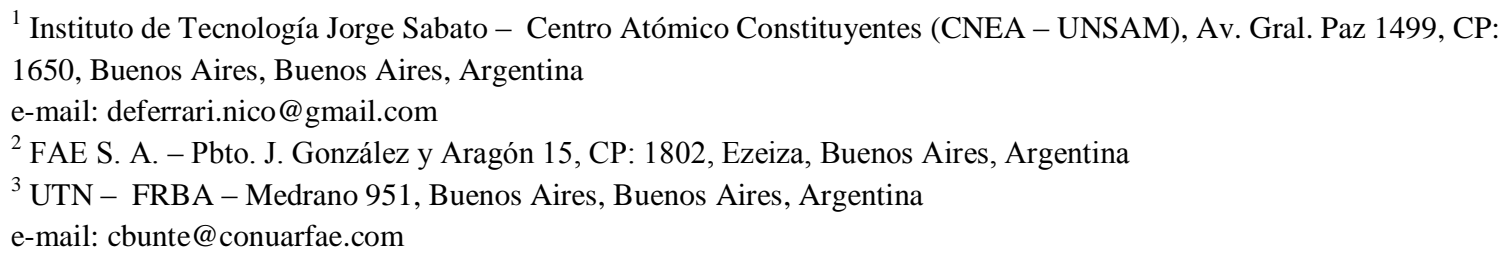

\section{RESUMEN}

En el desarrollo de procesos de conformado en caliente, los productos metálicos deben cumplir los requerimientos impuestos por las normas vigentes. En muchos casos, la microestructura requerida solo puede obtenerse a través del procesamiento termomecánico. Con el titanio y sus aleaciones, se presenta la dificultad adicional de estrechos rangos de formabilidad en caliente.

En este trabajo se estudió la formabilidad de distintas aleaciones de titanio de calidad aeronáutica a través de ensayos de compresión y torsión en caliente. Los resultados fueron utilizados para determinar el comportamiento del material, con el objeto de optimizar variables operativas en procesos de forja y extrusión, como temperatura de trabajo, velocidad de deformación, reducción de área, etc. Adicionalmente el ensayo de torsión en caliente permitió simular rutas de procesamiento y estudiar las microestructuras resultantes, las que fueron comparadas satisfactoriamente con las requeridas por las normas actuales. Al mismo tiempo se pudo determinar la cantidad necesaria de reducción en el último paso de forjado para obtener dicha microestructura. A partir de la información obtenida en los ensayos de formabilidad, y de los datos registrados en pruebas a escala real realizadas en Ti Gr 2, se simularon distintos procesos de extrusión por elementos finitos (FE). Para calibrar el modelo computacional fue también necesario medir la evolución de temperatura del material durante la alimentación de la prensa y evaluar el comportamiento de los lubricantes disponibles. Finalmente, el modelo permitió calcular con precisión la fuerza de extrusión requerida en el caso de los perfiles ensayados experimentalmente. De esta forma se pudo determinar la viabilidad de la extrusión de diferentes perfiles optimizando las variables de operación requeridas para mantener la presión de extrusión dentro de la capacidad operativa de la prensa y al mismo tiempo obtener las propiedades y microestructuras requeridas por las normas vigentes.

Palabras clave: formabilidad / trabajabilidad, extrusión, torsión en caliente, Ti-6Al-4V, procesamiento termomecánico.

\section{ABSTRACT}

In the development of hot metal forming, the metallic products must comply with the requirements imposed by current standards. In some cases, the required microstructure can only be obtained through thermomechanical processing. With titanium and its alloys, small ranges of good workability are an additional difficulty.

In this work the hot-workability of aeronautical quality titanium alloys was studied through hot torsion 
and compression testing. The results were used to define the material's plastic behaviour seeking to optimize forging and extrusion operating parameters, such as temperature, strain rate, area reduction, etc. Additionally, different processing routes were simulated through hot-torsion testing and the resulting microstructures were analysed and successfully compared to the current standards. At the same time, the minimum amount of area reduction required to obtain such microstructure was determined.

The results of hot-workability testing plus the information registered in full-scale Grade 2 Ti extrusion tests were used to simulate different extrusion processes with FEA. To calibrate the FE model it was also necessary to evaluate the billet's temperature evolution through the press's feeding system and the behaviour of the available lubricants. The FE model proved to be accurate to predict the required extrusion force for the tested geometries. This way, the feasibility of extruding different sections was determined and the operating parameters were optimized to maintain the extrusion pressure within operative limits as well as to obtain the material's properties and microstructures required in current standards.

Keywords: workability, extrusion, hot-torsion, Ti-6Al-4V, thermomechanical processes.

\section{INTRODUCCIÓN}

\subsection{Objetivo}

El objetivo de este trabajo es estudiar la trabajabilidad en caliente de Ti CP Gr 2 y Ti-6Al-4V a través del ensayo de torsión. En particular se espera poder determinar las curvas de fluencia y ductilidad, las cuales pueden ajustarse a distintos modelos matemáticos que permiten interpolar y extrapolar curvas de fluencia a condiciones diferentes a las ensayadas. Para hacer esto va a ser necesario relevar el modo de funcionamiento del equipo y, eventualmente, realizar modificaciones.

También se busca determinar las microestructuras resultantes de distintas simulaciones de procesos termomecánicos para compararlas con las normas vigentes y verificar su aceptación o rechazo.

En simultáneo se modeló conjuntamente con ABATE y DEFERRARI [1] un proceso de extrusión de Ti CP Gr 2 en un software de elementos finitos (FE) a partir de los datos obtenidos de una prueba a escala real realizada en planta. El objetivo es que, en un futuro, los resultados puedan utilizarse para ajustar y validar el modelo, lo que tendrá aplicación inmediata en la optimización de los procesos de extrusión a escala industrial.

\subsection{Procesamiento de las aleaciones de titanio}

El procesamiento primario es fundamental para definir las propiedades finales del producto, en particular, en casos donde el procesamiento secundario no aporte suficiente deformación como para modificar notablemente las propiedades del producto.

En el caso del titanio, la reducción primaria del lingote se realiza exclusivamente en el campo de la fase $\beta$, es decir, por sobre la temperatura $\beta$-transus. Esto responde principalmente a la mayor ductilidad de la fase BCC y a la muy fuerte reducción de la resistencia mecánica a partir del cambio de fase. De esta forma se reduce la energía necesaria para procesar el material y al mismo tiempo la cantidad de defectos que pueden producirse.

Posteriormente, las etapas de reducción adicionales se realizan normalmente debajo de $\beta$-transus y son esenciales para el refinamiento de la estructura. Además del trabajado mecánico es posible refinar la estructura aún más mediante la utilización de un hipertemple o temple de solubilización [2]. Este tipo de tratamiento es de uso frecuente en aleaciones $\alpha+\beta$ previo a la reducción final en el campo $\alpha+\beta$ [3].

El grado de deformación o reducción en el campo bifásico guarda estricta relación con el refinamiento de la estructura y a su vez con las propiedades mecánicas finales del producto (especialmente la resistencia mecánica y la ductilidad) $[2,3,4]$.

En los semielaborados producidos con el objeto de ser forjados posteriormente, la reducción del tamaño de grano es fundamental para mejorar su trabajabilidad y disminuir la aparición de defectos. En aleaciones bifásicas, ambas fases deben refinarse. El refinamiento de la fase $\beta$ se produce durante la primer reducción del lingote en fase $\beta$ y/o durante la recristalización en el hipertemple, mientras que los granos de $\alpha$ se refinan durante el procesado en el rango $\alpha+\beta$ [3].

Todas las aleaciones de titanio se caracterizan por tener un acotado rango de temperaturas de buena trabajabilidad. La $\beta$-transus limita la temperatura superior de trabajado en rango $\alpha+\beta$ por la necesidad de evitar el cambio de fase y la recristalización, que hecha por tierra la deformación acumulada en el rango bifá- 
sico y reduce drásticamente la ductilidad del producto final. Por su parte, el límite inferior está acotado en casi todos los casos por el endurecimiento por deformación y la aparición de fisuras superficiales. La fuerte variación de la resistencia a la deformación con la temperatura juega también un papel importante por su efecto en la energía necesaria para deformar el material.

\subsection{Relación entre el procesamiento, la microestructura y las propiedades}

El titanio y sus aleaciones se caracterizan por tener una muy marcada relación entre sus propiedades mecánicas y su microestructura. Indirectamente, su versatilidad puede atribuirse a la posibilidad de obtener un rango muy amplio de microestructuras con una cantidad acotada de aleaciones, y en consecuencia, abarcar un amplio espectro de propiedades mecánicas. Así como las propiedades mecánicas van estrictamente de la mano de la microestructura, esta última es un resultado directo de la composición de la aleación, su procesamiento y el tratamiento térmico posterior [2]. El factor clave en lo que hace a las etapas de procesamiento de las aleaciones de titanio es el control de la cinética de la transformación $\beta$ / $\alpha+\beta$ durante las distintas etapas de calentamiento y enfriamiento, para así poder controlar el tipo de estructuras presentes y su morfología.

\subsection{Trabajabilidad en caliente}

La trabajabilidad en caliente de un material se define como su capacidad para ser deformado a temperaturas elevadas, más precisamente cuando $\mathrm{T}>0.6 \cdot \mathrm{T}_{\text {fusión }}$. Por lo general, esta temperatura indica implícitamente que los procesos de deformación se realizan en condiciones donde los mecanismos de restauración dinámica (recuperación y/o recristalización) ocurren simultáneamente con la deformación [5,6,7].

La posibilidad de deformar plásticamente un material se encuentra por lo general limitada por la aparición de fisuras, defectos, etc. Cuando un material tiene buena trabajabilidad, es posible deformarlo más y producir formas más complejas antes que se produzca la fractura. La trabajabilidad de un material también se puede definir por la capacidad para obtener mejores terminaciones superficiales o mejores tolerancias.

El análisis de la trabajabilidad de un material implica el estudio de su resistencia mecánica y su ductilidad, y de la forma que estas características interactúan entre sí cuando se lo procesa mecánicamente. En otras palabras, una completa descripción de la trabajabilidad de un material se especifica por la dependencia que tienen su tensión de fluencia y ductilidad con las variables del proceso de conformado y con las variables metalúrgicas [7]. Entre las primeras, las más importantes son la deformación, la velocidad de deformación, la temperatura de conformado y los ciclos térmicos anteriores y posteriores. Entre las segundas se destacan la microestructura, los mecanismos de fractura, las transformaciones de fase, etc.

Resulta evidente la estrecha relación entre el concepto de trabajabilidad y el diseño de los procesos de conformado. El estudio de la trabajabilidad del material en los rangos operativos del proceso es fundamental para obtener productos que cumplan con los requisitos de calidad requeridos (óptimas propiedades mecánicas, microestructuras adecuadas, tolerancias dimensionales, prevención de defectos, alta productividad, etc.).

\subsection{Ensayo de torsión en caliente}

En el ensayo de torsión las probetas son deformadas en un estado de corte puro por la acción de un momento torsor. A diferencia de los ensayos mecánicos más sencillos, permite aplicar grandes deformaciones sin cambios apreciables en la geometría de la probeta, y a velocidad de deformación constante. La limitación más evidente del ensayo de tracción es el fenómeno de estricción, que no permite desarrollar las grandes deformaciones que caracterizan a los procesos de conformado en caliente como forja o extrusión. El ensayo de torsión es muy utilizado para evaluar la trabajabilidad de los materiales, especialmente en caliente, en donde la ventaja de alcanzar grandes deformaciones es más evidente.

Los factores que distinguen al ensayo de torsión sobre otros ensayos son entonces:

- La posibilidad de deformar severamente el material de forma homogénea y a velocidades de deformación superficial constante, sin el inconveniente de los ensayos de compresión que están sujetos a inhomogeneidad en la deformación por efecto de la fricción.

- La posibilidad de aplicar deformaciones torsionales muy grandes manteniendo la geometría de la probeta prácticamente constante hasta la rotura.

- La posibilidad de obtener información sobre la resistencia a la deformación, la ductilidad, los procesos de recuperación y recristalización y los mecanismos de fractura o localización de la deformación.

En consecuencia, además de estudiar el comportamiento mecánico del material, es posible también realizar fácilmente programas de deformación (simulaciones termomecánicas). En estos casos se aplican dis- 
tintas etapas de deformación en forma sucesiva, cada una a velocidad de deformación y temperatura independientes.

\section{MATERIALES Y MÉTODOS}

\subsection{Máquina de torsión en caliente}

La máquina utilizada en este trabajo fue desarrollada ad hoc hace aproximadamente 20 años por el Instituto Argentino de Siderurgia (IAS) en San Nicolás, Prov. de Buenos Aires. El software, también desarrollado en el IAS, permite llevar a cabo dos tipos distintos de ensayos: ensayos a rotura a velocidades de deformación y temperatura constante, o bien secuencias de deformación por etapas (simulación termomecánica) (Figura 1). El primer tipo de ensayo permite estudiar la resistencia del material y su ductilidad. El segundo permite estudiar la microestructura resultante luego de un la secuencia de deformación y también la resistencia del material en las sucesivas etapas.

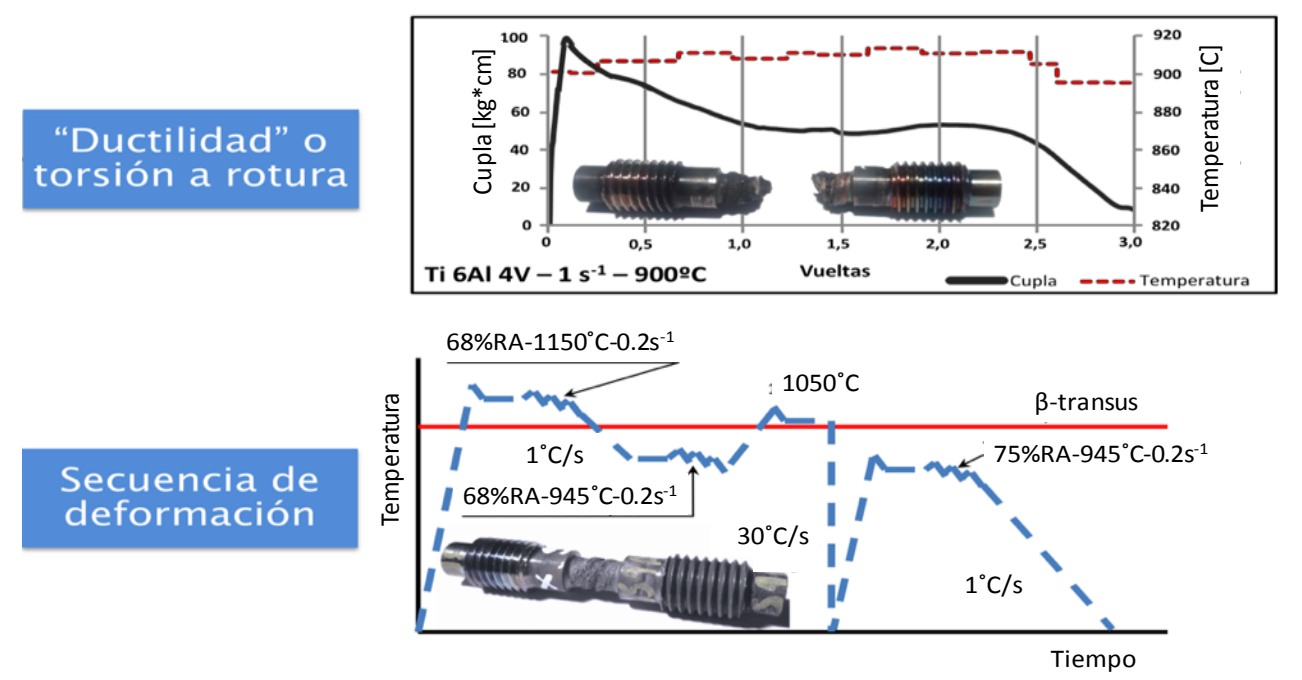

Figura 1: Formas de uso de la máquina de torsión en caliente.

\subsection{Probetas}

En este trabajo se utilizaron probetas roscadas en ambos extremos con una sección calibrada de $7 \mathrm{~mm}$ de diámetro y $7 \mathrm{~mm}$ de longitud. La elección de las dimensiones de la probeta depende exclusivamente del diseño de la máquina y a la experiencia del operador de la misma. Por el momento no existe estandarización formal en cuanto a dichas dimensiones [6].

Los ensayos se realizaron sin desplazamiento axial de la mordaza fija, como la mayoría de los ensayos descriptos en la bibliografía. Distintos autores han demostrado que las tensiones axiales resultantes de esto pueden despreciarse frente a las tensiones generadas por torsión de la probeta $[6,8]$.

Una vez ensayadas las probetas se extrajeron muestras para analizarlas al microscopio. Se realizaron tres cortes a fin de obtener una sección longitudinal de un tamaño sencillo de manipular (Figura 5). Los cortes se realizaron en una fresadora universal con una sierra circular de $1 \mathrm{~mm}$ de espesor lubricada con aceite de corte. El corte se realizó descentrado sobre la probeta de tal forma de dejar expuesta la zona central.

Todas las muestras fueron desbastadas hasta papel de grano $1500 \mathrm{y}$ posteriormente pulidas con ceniza de dicromato de amonio disuelta en una solución de HF de pureza comercial al $0.5 \%$. El ataque se realizó durante $5 \mathrm{~s}$ con un reactivo de uso general para aleaciones de titanio compuesto por $85 \% \mathrm{H}_{2} \mathrm{O}+10 \% \mathrm{HF}+$ $5 \% \mathrm{HNO}_{3}$.

\subsection{Material}

El material disponible para los ensayos fue fabricado in-house por FAE S.A. La empresa cuenta con un horno de fusión al vacío que permite producir lingotes de titanio de hasta $1500 \mathrm{~kg}$. Los lingotes de obtienen por el método de doble fusión o refusión al vacío. La composición química se detalla en la tabla 1.

De los lingotes disponibles (Ti-6Al-4V y Ti CP Gr 2) se cortaron rodajas de $20 \mathrm{~mm}$ de espesor, las 
cuales se cortaron nuevamente de forma transversal para obtener barras de sección cuadrada de $20 \mathrm{~mm}$ de lado. Las probetas fueron obtenidas por mecanizado de estas barras en un torno de control numérico.

En el caso de las probetas obtenidas de barras forjadas (Ti-6Al-4V), el método de extracción fue similar, pero con la diferencia de que las rodajas fueron obtenidas de una barra previamente forjada en caliente de $130 \mathrm{~mm}$ de diámetro (Reducción de Área $68 \%, 950{ }^{\circ} \mathrm{C}$ ).

Tabla 1: Composición química de los materiales.

\begin{tabular}{l|c|c|c|c|c|c|c|c|c|c|c|c|c|c|c}
\hline & $\begin{array}{c}\mathbf{A l} \\
{[\%]}\end{array}$ & $\begin{array}{c}\mathbf{V} \\
{[\%]}\end{array}$ & $\begin{array}{c}\mathbf{F e} \\
{[\%]}\end{array}$ & $\begin{array}{c}\mathbf{C} \\
{[\%]}\end{array}$ & $\begin{array}{c}\mathbf{H} \\
{[\%]}\end{array}$ & $\begin{array}{c}\mathbf{O} \\
{[\%]}\end{array}$ & $\begin{array}{c}\mathbf{N} \\
{[\%]}\end{array}$ & $\begin{array}{c}\mathbf{C u} \\
{[\%]}\end{array}$ & $\begin{array}{c}\text { Mo } \\
{[\%]}\end{array}$ & $\begin{array}{c}\text { Si } \\
{[\%]}\end{array}$ & $\begin{array}{c}\mathbf{Z r} \\
{[\%]}\end{array}$ & $\begin{array}{c}\mathbf{P} \\
{[\%]}\end{array}$ & $\begin{array}{c}\mathbf{C r} \\
{[\%]}\end{array}$ & $\begin{array}{c}\text { OTROS } \\
{[\%]}\end{array}$ & Ti [\%] \\
\hline Ti-6Al-4V & 6.09 & 3.54 & .093 & .018 & .0037 & .157 & .005 & .004 & .001 & .023 & .0009 & .003 & & .356 & \\
\hline & & & & & & & & & & & 6 & .21 & & balance \\
\hline Ti CP Gr2 & .016 & .093 & .144 & .019 & .0010 & .202 & .002 & .001 & .003 & .054 & .0012 & .003 & & .281 & balance \\
\hline
\end{tabular}

Cuando se utilizaron probetas mecanizadas a partir de un lingote, la microestructura de partida fue la resultante del proceso de colado (estructura de colada). Desde el punto de vista tecnológico, ensayar probetas con este tipo de estructura es útil para conocer el comportamiento del material en las etapas de trabajado primario que involucren el forjado en rango $\beta$, donde se produce la "rotura de la estructura de colada".

A las temperaturas de trabajado sub-transus, que son utilizadas en operaciones de forja intermedia o final, no es de gran interés práctico ensayar probetas con estructura de colada. La razón de esto es que los semielaborados utilizados industrialmente en estas operaciones son siempre trabajados previamente en rango $\beta$. Al no contar con probetas de Ti CP Gr 2 extraídas de barras forjadas por encima de $\beta$-transus, se decidió incluir una etapa previa de simulación termomecánica en los ensayos a rotura realizados a temperaturas subtransus. Esta etapa previa representa de forma simplificada una típica reducción primaria de un lingote por forja libre. En función de esto se relevaron los parámetros de las operaciones normalmente utilizadas en planta para la forja de lingotes y se aplicaron en dicha etapa previa al ensayo de torsión a rotura.

El ajuste del modelo de elementos finitos se realizó en conjunto con [1] a partir del software Simufact.Forming, en el laboratorio de Procesos de Mecanizado y Conformado de INTI-Mecánica. Para ajustar los datos de pre procesamiento se utilizaron los resultados de los ensayos de torsión en caliente. Para verificar los coeficientes de transmisión de calor (HTC) y la emisividad se realizaron ensayos de enfriamiento. Para esto último se realizó el calentamiento de un billet de $184 \mathrm{~mm}$ de altura, $170 \mathrm{~mm}$ de diámetro externo y $68 \mathrm{~mm}$ de diámetro interno hasta $1030{ }^{\circ} \mathrm{C}$. Una vez fuera del horno se colocaron 6 termocuplas en distintas posiciones.

\subsection{Pruebas de extrusión en planta}

Como primer etapa en la validación del modelo se relevaron los datos de una prueba de extrusión realizada en planta. En la prueba piloto se obtuvieron 3 diferentes productos extrudados en Ti CP Gr 2:

- Barra maciza de $52 \mathrm{~mm}$ de diámetro a partir de un billet de $170 \mathrm{~mm}$ de diámetro.

- Tubo de $65 \mathrm{~mm}$ de diámetro exterior y $40 \mathrm{~mm}$ de diámetro interior (65x40), a partir de un billet perforado de $170 \mathrm{~mm}$ de diámetro externo y $65 \mathrm{~mm}$ de diámetro interno.

- Tubo de $65 \mathrm{~mm}$ de diámetro exterior x $55 \mathrm{~mm}$ de diámetro interior (65x55), a partir de un billet perforado de $170 \mathrm{~mm}$ de diámetro externo y $65 \mathrm{~mm}$ de diámetro interno.

Se utilizó una prensa hidráulica horizontal de 1600 t de capacidad nominal; con una velocidad de desplazamiento de $40 \mathrm{~mm} / \mathrm{s}$. El cilindro contenedor del material posee un diámetro interno de $175 \mathrm{~mm}$, con un sistema de precalentamiento por resistencia eléctrica. El calentamiento de los billets fue realizado por un horno de inducción horizontal continuo y la temperatura a la salida del horno fue medida con un pirómetro óptico. Como lubricante se utilizó grafito en solución acuosa aplicado directamente sobre la matriz con estopa de lana.

\subsection{Ensayos de torsión}

El Ti CP Gr 2 fue ensayado a cuatro temperaturas, tres sub-transus $\left(700{ }^{\circ} \mathrm{C}, 760{ }^{\circ} \mathrm{C}, 840{ }^{\circ} \mathrm{C}\right)$ y una en el campo $\beta\left(960{ }^{\circ} \mathrm{C}\right)$; mientras que para Ti-6Al-4V se seleccionaron dos temperaturas en el campo $\alpha+\beta\left(900{ }^{\circ} \mathrm{C}, 950\right.$ ${ }^{\circ} \mathrm{C}$ ) y una en el campo $\beta\left(1150{ }^{\circ} \mathrm{C}\right)$. En todos los casos se mantuvo la probeta a la temperatura prevista para el ensayo durante 4 min previos a la deformación. Este tiempo es suficiente para garantizar la homogeneidad térmica y, según FROES, para la finalización de la transformación de fase $\beta$ [3]. 
Para cada temperatura se realizaron ensayos a cuatro velocidades de deformación equivalente $\left(0.1 \mathrm{~s}^{-1}\right.$, $1 \mathrm{~s}^{-1}, 10 \mathrm{~s}^{-1}, 35 \mathrm{~s}^{-1}$ ) (tabla 2). Esto permite determinar el comportamiento del material en el amplio rango de velocidades de deformación características de los procesos de forja y, en especial, de extrusión.

Tabla 2: Resumen de ensayos de torsión a rotura.

\begin{tabular}{c|l|c|c|c|c}
\hline \multirow{2}{*}{ ALEACIÓN } & \multirow{2}{*}{ ESTADO MET. } & T' & TRABAJADO & \multicolumn{4}{|c}{$\mathbf{T}^{\circ}$ TRABAJADO EN $\boldsymbol{\alpha}+\boldsymbol{\beta}\left[^{\circ} \mathbf{C}\right.$ ] } \\
\cline { 4 - 6 } & & EN $\beta\left[{ }^{\circ} \mathbf{C}\right]$ & $\mathbf{1}$ & $\mathbf{2}$ & $\mathbf{3}$ \\
\hline \multirow{2}{*}{ Ti CP Gr 2} & Lingote & 960 & 840 & 760 & 700 \\
\hline \multirow{2}{*}{ Ti-6Al-4V } & Lingote & 1150 & 950 & & \\
\cline { 5 - 7 } & Barra Forjada & & 950 & 900 & \\
\hline
\end{tabular}

\section{RESULTADOS Y DISCUSIÓN}

\subsection{Curvas de momento torsor y curvas de fluencia}

Se observó en algunos casos que las cuplas de los ensayos realizados a $35 \mathrm{~s}^{-1}$ fueron menores a las de los ensayos a $10 \mathrm{~s}^{-1}$. Se supone que podría resultar de la manifestación de procesos de inestabilidad plástica, aunque no se cuenta con la cantidad de ensayos necesarios para asegurarlo. Queda pendiente su análisis para futuras ampliaciones del trabajo. Se decidió no considerarlos en el cálculo de la sensibilidad a la velocidad de deformación (m). De igual manera se observa este mismo comportamiento pero en menor medida en las curvas de

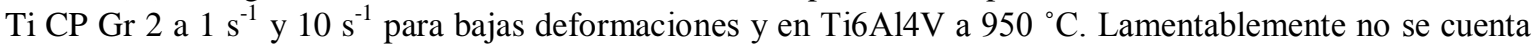
con mayor cantidad de ensayos que permitan reducir la incidencia de pequeñas variaciones y heterogeneidades en los materiales de partida, especialmente los obtenidos de lingotes en estado de colada. La repetición de los ensayos a futuro y el diseño de una nueva máquina son posibilidad de medir esfuerzos axiales y torsionales descompuestos en otros ejes permitirán identificar estas desviaciones y considerar su aparición en los cálculos. Salvo por los casos mencionados, resulta claro que la cupla máxima desciende con el aumento de la temperatura y aumenta con el aumento en la velocidad de deformación. Las curvas de momento torsor (Figura 2) se convirtieron en curvas de fluencia (Figuras 3 y 4) a partir del formulismo desarrollado por FIELDS y BACKOFEN [9].
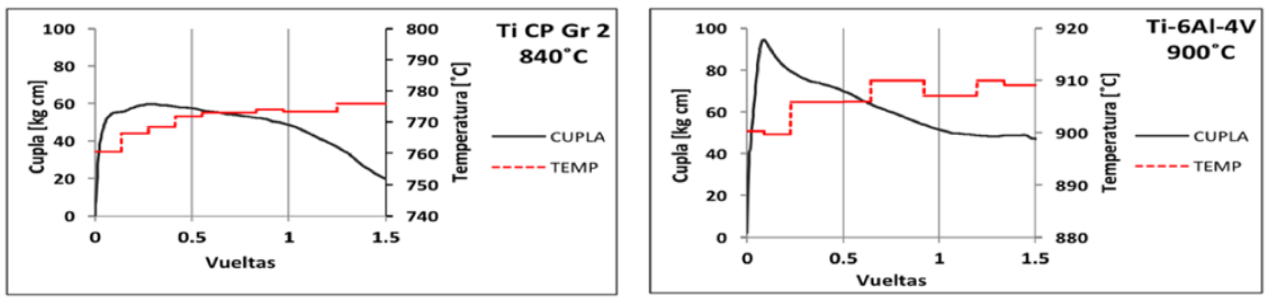

Figura 2: Curvas de torsión (Ti CP Gr 2 y Ti-6Al-4V) 

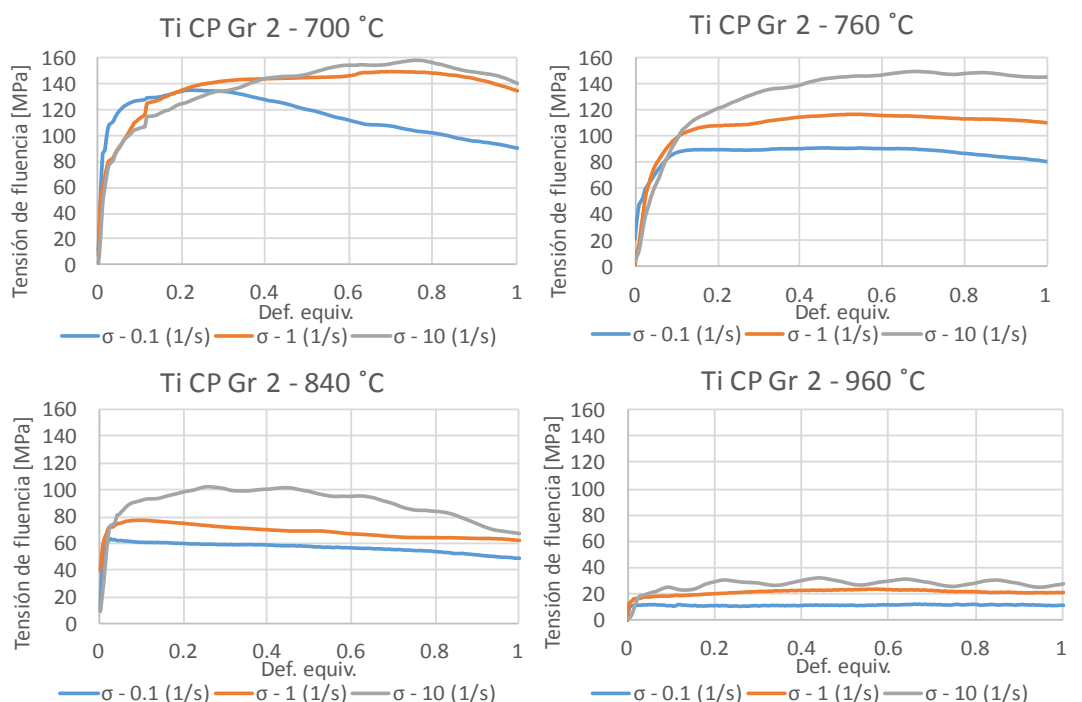

Figura 3: Curvas de fluencia Ti CP Gr 2.

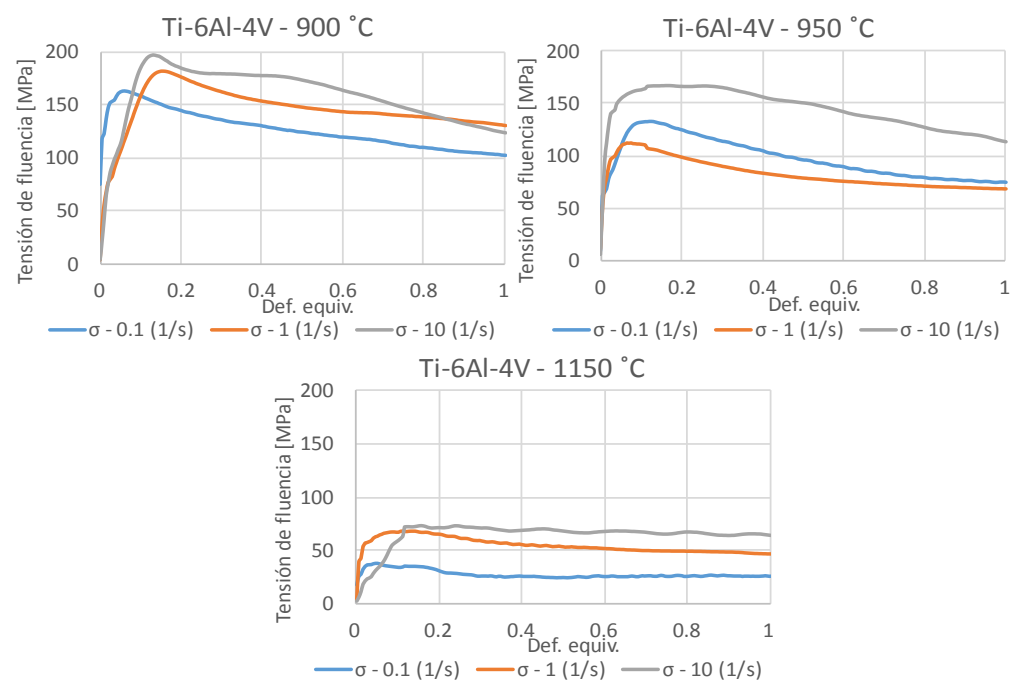

Figura 4: Curvas de fluencia Ti-6Al-4V.

A partir de las curvas de fluencia se observa que la resistencia a la deformación de la fase $\beta$ es entre 5 y 10 veces menor que la de la fase $\alpha / \alpha+\beta$ en Ti CP Gr 2 y Ti-6Al-4V. Esta gran diferencia es un factor muy importante en los procesos industriales de conformado plástico. Pequeños enfriamientos durante o antes de la deformación, capaces de llevar al material por debajo de $\beta$-transus, producen a un aumento en la resistencia a la deformación de un orden de magnitud. Si esto sucede, la capacidad máxima del equipamiento puede ser fácilmente sobrepasada, pudiendo generarse roturas o accidentes.

\subsection{Micro y Macroestructura de una probeta de torsión en caliente}

Una probeta sometida a un ensayo de torsión guarda mucha información sobre la evolución microestructural del material. Existen distintas zonas a lo largo de la probeta, que si bien son sometidas a casi el mismo ciclo térmico, permiten observar la microestructura resultante de distintos grados de deformación.

De esta forma es posible identificar las distintas zonas de la probeta, que como ya se explicó, están sometidas a prácticamente el mismo ciclo térmico pero a muy diferentes condiciones de trabajado mecánico:

- Zonas sin deformación: roscas y hombros.

- Interfases entre zonas deformadas y zonas sin deformación: radios de acuerdo y eje neutro central. En este último caso no es siempre posible observar dicha transición, dependerá de la sensibilidad de la estructura a pequeñas deformaciones. 
- Zonas deformadas: zona calibrada de la probeta a excepción del eje neutro.

En la Figura 5 se muestra una macrografía de una probeta torsionada de Ti-6Al-4V previamente forjado en donde se distinguen los tres tipos de zonas. Simplemente observando el tamaño de grano, resulta evidente que existen dos zonas deformadas, cada una en la parte externa de la zona calibrada. La deformación es homogénea a lo largo del eje longitudinal de la zona calibrada y aumenta linealmente con el radio, siendo nula sobre el eje neutro central y máxima sobre la superficie. La velocidad de deformación también tiene este comportamiento. Esta información se puede ver en detalle en las micrografías de la Figura 6.

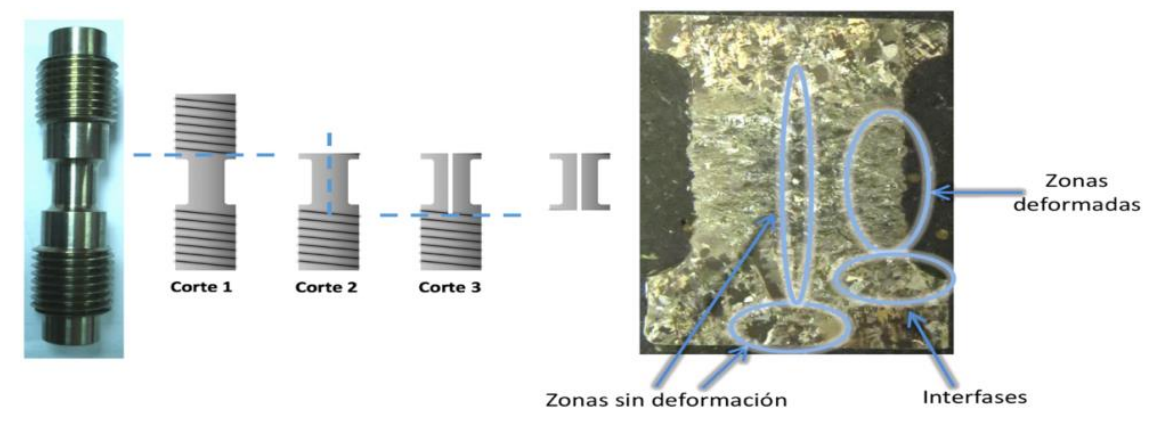

Figura 5: Cortes de probeta de torsión y zonas de deformación en probeta torsionada.

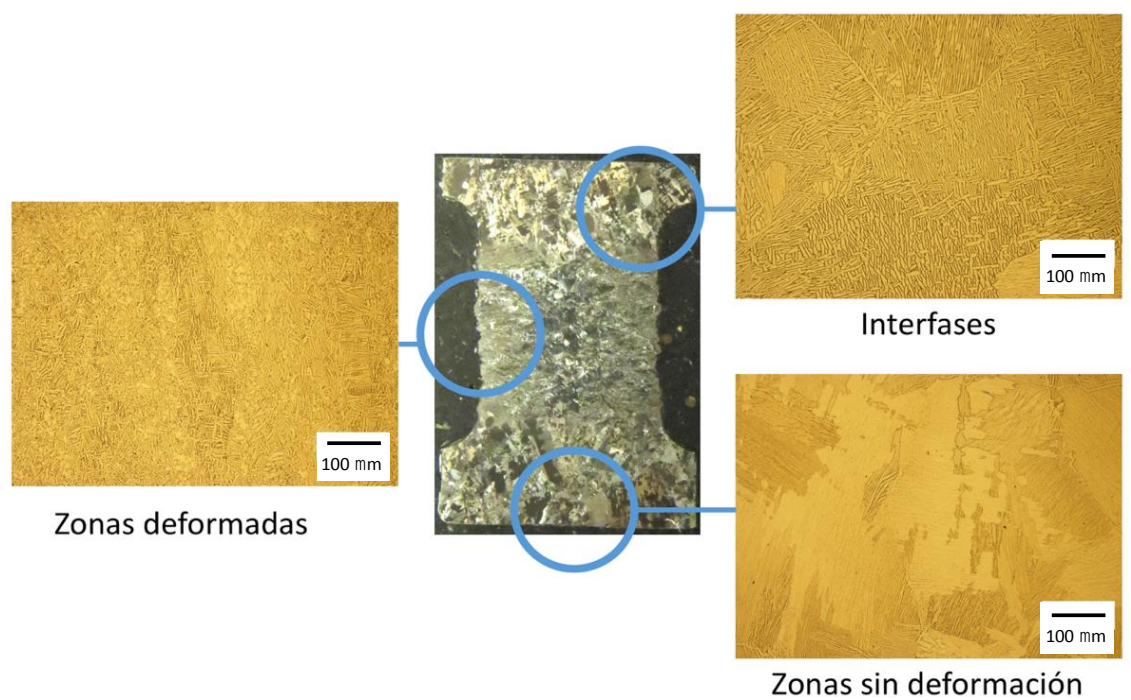

Figura 6: Micrografías de una probeta de torsión luego del ensayo.

\subsection{Simulación por torsión en caliente de un proceso de forja}

En este trabajo se simularon tres rutas de procesamiento en probetas de Ti-6Al-4V con estructura de colada y con estructura acicular resultante del forjado del lingote en rango $\beta$ seguido de un templado ( $\beta$-quenching). Las rutas para las probetas extraídas de lingotes incluyeron como primer etapa la simulación del forjado en rango $\beta$, mientras que las probetas de estructura forjada iniciaron la simulación a partir de la primera etapa de deformación en rango $\alpha+\beta$. En la Figura 7 se detallan las rutas de procesamiento. Al igual que para los ensayos de torsión a la rotura, los tiempos de homogenización térmica y estructural fueron de 4 min. En todos los casos se utilizaron deformaciones, velocidades de deformación y temperaturas correspondientes a los valores habituales utilizados en la industria y coherentes con lo que se establece en los mapas de procesamiento desarrollados por PRASAD et al. [10,11]. 

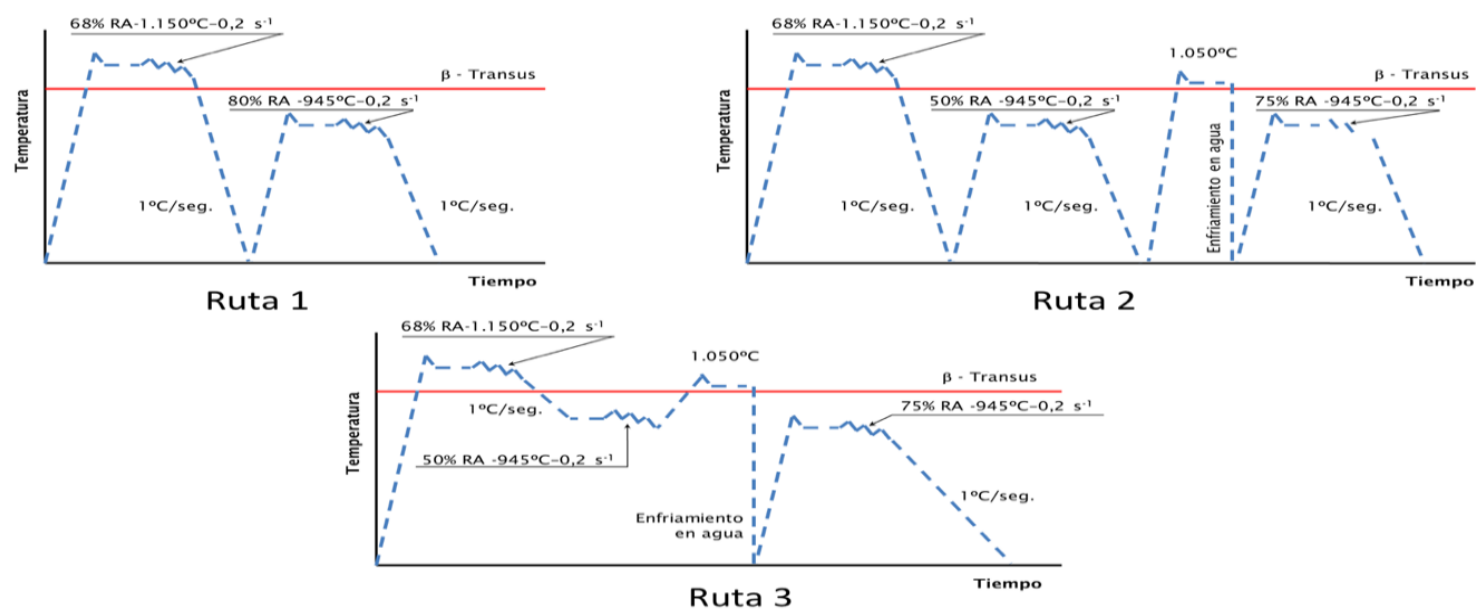

Figura 7: Diagramas de simulación de forjado para Ti-6Al-4V.

El objetivo de estas simulaciones fue determinar la microestructura resultante y verificar el cumplimiento de las normas ETTC-2 [12] e ISO 20160:2006 [13] que establecen las microestructuras aceptadas para distintos productos de titanio.

En la Figura 8 se observan las macro y microestructuras de partida para Ti-6Al-4V, obtenidas directamente del lingote y barra forjada. A simple vista se observa en el material forjado una estructura mucho más fina y acicular, similar a lo que en estas aleaciones resulta de transformaciones de tipo Widmanstätten, característica del enfriamiento rápido desde el campo $\beta$, como se observa en la Figura 9 y 10.
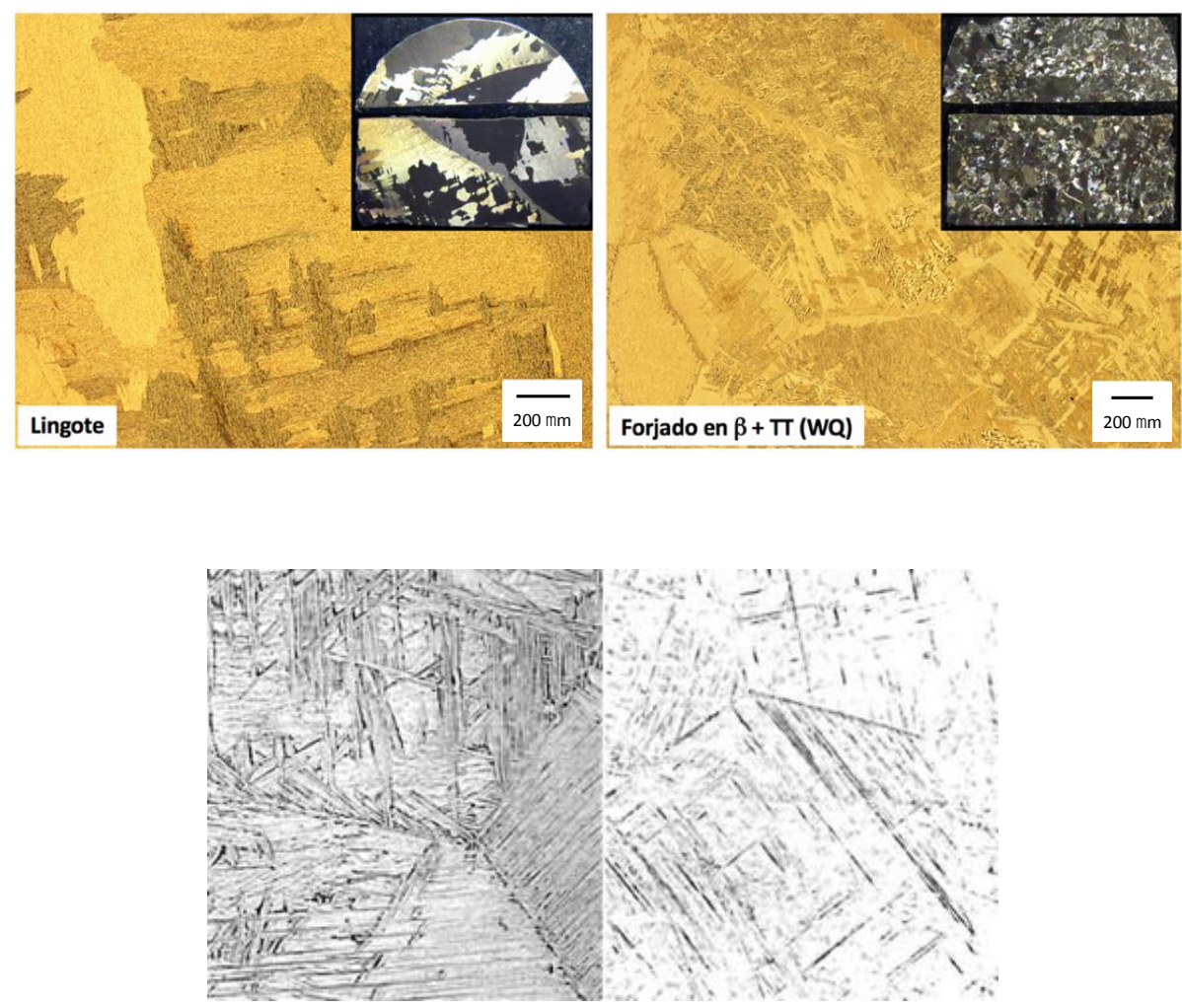

Figura 8: Estructuras de partida Ti-6Al-4V. 


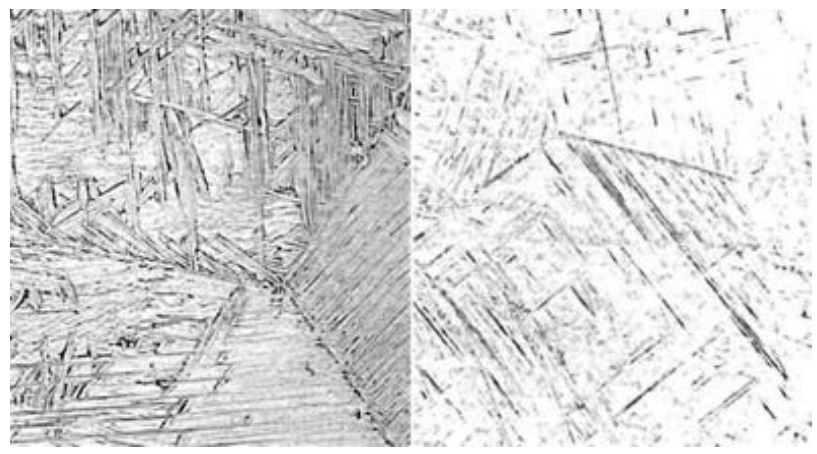

Figura 9: Microestructuras obtenidas por enfriamiento rápido en Ti-6Al-4V. Widmastätten (izq). Martensita (der) [3].

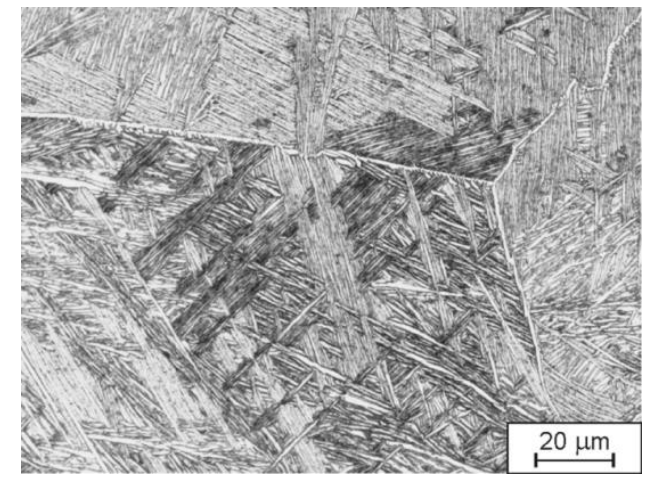

Figura 10: Estructura acicular tipo canasta o Widmästatten obtenida en Ti-6Al-4V por enfriamiento desde $\beta$ a $9{ }^{\circ} \mathrm{C} / \mathrm{s}$ [14].

En la Figura 11 se observan las macro y microestructuras resultantes de la simulación 1 para una probeta de Ti-6Al-4V obtenida de barra forjada, previo y post recocido. La microestructura de partida para este caso es la que se observa en la Figura 8 (derecha). Se realizó únicamente la etapa de deformación en rango $\alpha+\beta$ tal como fue descripto en el párrafo anterior, y se puede verificar que no queda evidencia de la estructura acicular previa, lo cual indica que la misma fue satisfactoriamente trabajada para dar lugar a una estructura equiaxiada luego del recocido (Figura 9 - derecha). Luego, en la Figura 12 se observan las microestructuras obtenidas para las 3 rutas de procesamiento simuladas a partir de probetas extraídas de lingote. También se detalla una de las microestructuras aceptadas para productos de calidad superior a la standard por la norma ETTC-2 [12]. En los 6 casos (3 rutas de procesamiento para 2 materiales de partida, lingote y forjado) se obtuvieron microestructuras aceptadas por las normas para productos de calidad superior a la standard.
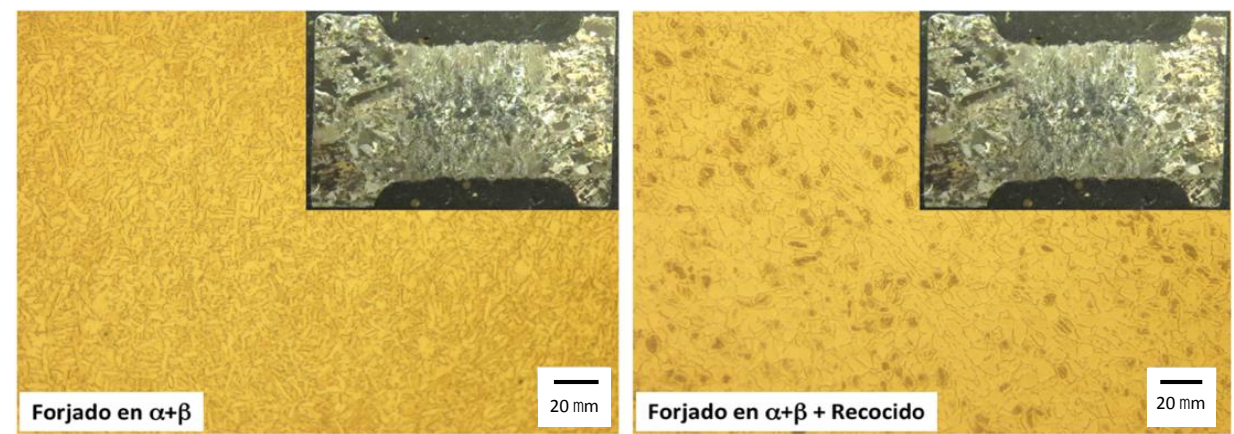

Figura 11: Micrografías obtenidas de la simulación 1 en probetas de Ti-6Al-4V forjado. 


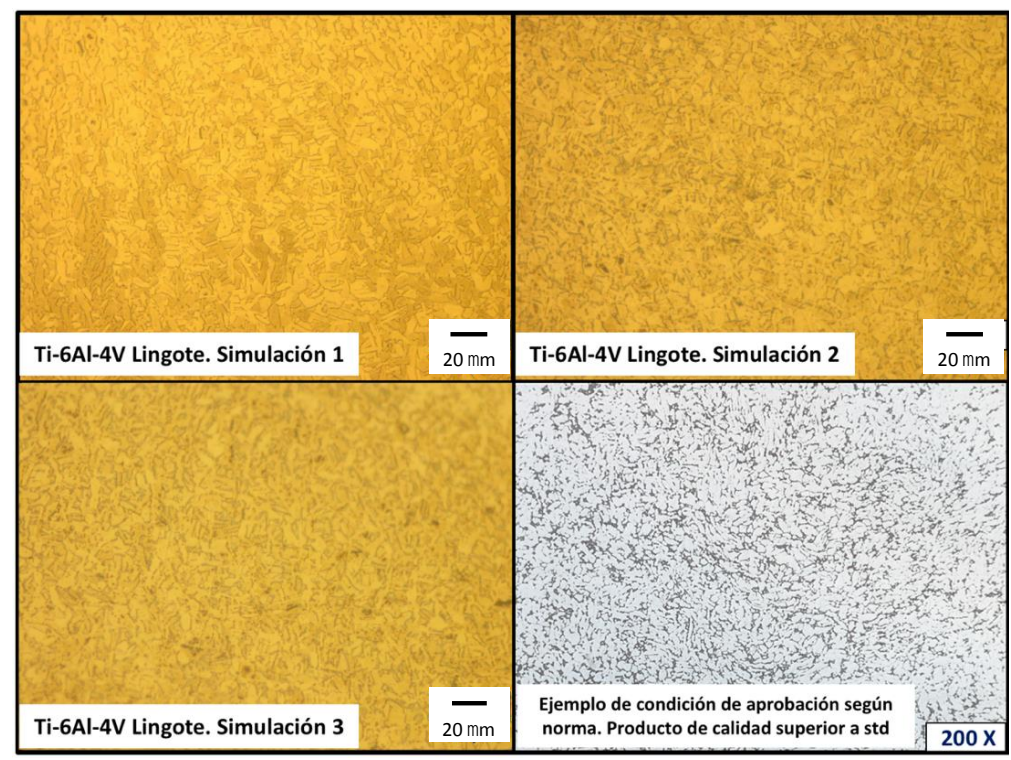

Figura 12: Micrografías resultantes de las simulaciones luego del recocido y ejemplo de norma ETTC-2.

\subsection{Efecto de la cantidad de deformación en rango $\alpha+\beta$}

Normalmente se asume que para Ti-6Al-4V es necesario al menos un $50 \%$ de reducción de área en el campo $\alpha+\beta$ para romper la estructura acicular de solidificación producida por el enfriamiento desde el campo $\beta$ $[2,3,4]$. Para observar esto se realizaron distintos ensayos de torsión y de compresión.

A partir de la secuencia de simulación 1 se realizaron simulaciones similares con cantidades decrecientes de reducción en el campo $\alpha+\beta$. En la Figura 13 se observan las microestructuras resultantes de cada ensayo.

Cuando la deformación en la zona bifásica es alta $(60 \%)$ se observa que la microestructura resultante luego del recocido es de granos poligonales predominantemente equiaxiados. A menores deformaciones (40\%) se observan pequeñas zonas de granos equiaxiados y otras en donde la deformación es heterogénea y los granos son mayoritariamente alargados. Esto indica que la deformación fue insuficiente y que la estructura conserva la morfología resultante del enfriamiento desde rango $\beta$. A deformaciones bajas (20\%) coexisten distintas morfologías, evidencia de una microestructura sumamente heterogénea con evidencia de las estructuras previamente formadas durante el enfriamiento desde temperaturas superiores a $\beta$-transus.

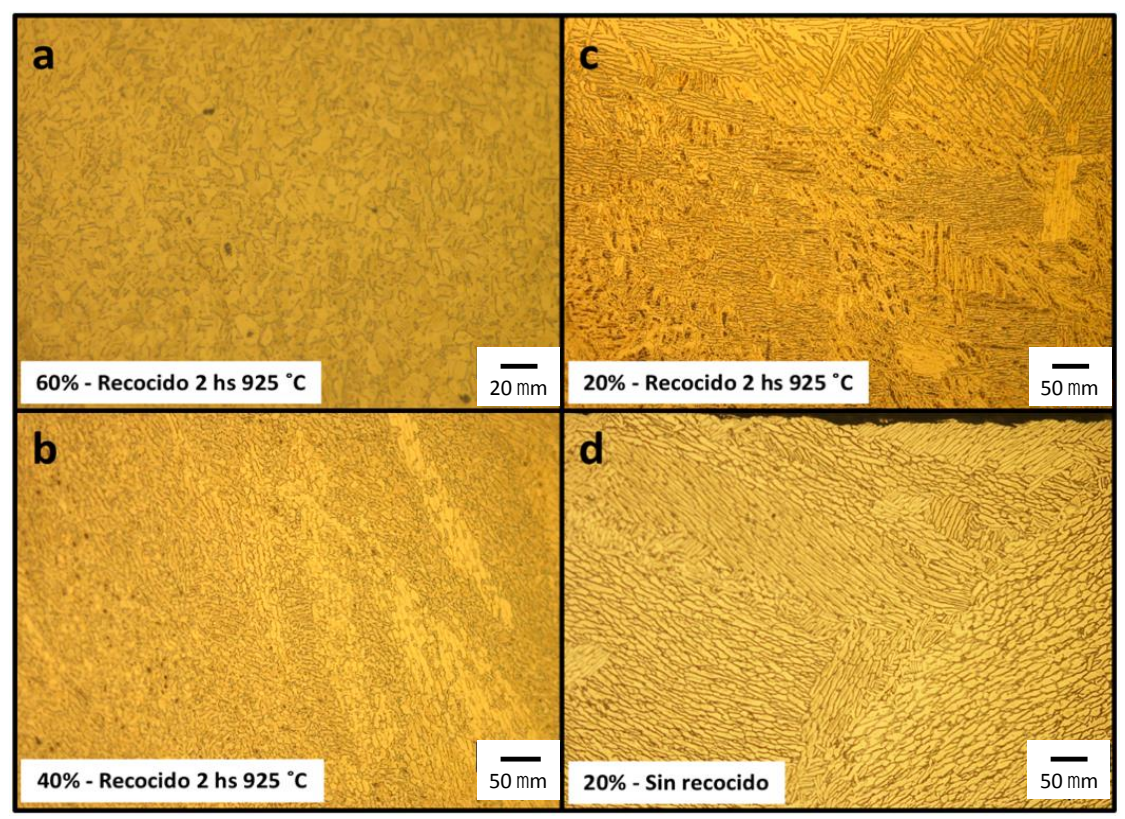

Figura 13: Efecto de la cantidad de deformación en campo $\alpha+\beta$ sobre la microestructura. Ti-6Al-4V. 
Este mismo tipo de observación se realizó también a través del ensayo de compresión con probetas con forma de cuña, y en ambos casos se estima que la cantidad de reducción en campo $\alpha+\beta$ debe superar el 50-60\% para obtener una estructura satisfactoriamente equiaxiada y homogénea luego del recocido.

\subsection{Ajuste y reproducción de procesos de extrusión por elementos finitos (Ti CP Gr 2)}

Para la validación por simulación numérica se realizó un modelo de enfriamiento al aire, tomando como condición de partida una temperatura del material de $1000{ }^{\circ} \mathrm{C}$. El ajuste de la curva obtenida por simulación se realizó por iteración con un HTC pieza-ambiente variable con la temperatura. Esto permitió representar correctamente la liberación de calor que se produce a $882{ }^{\circ} \mathrm{C}$ como resultado del cambio de fase que presenta el Ti CP Gr 2. La curva de enfriamiento experimental y la obtenida por simulación en la posición correspondiente a la termocupla 1 pueden verse en la Figura 14.

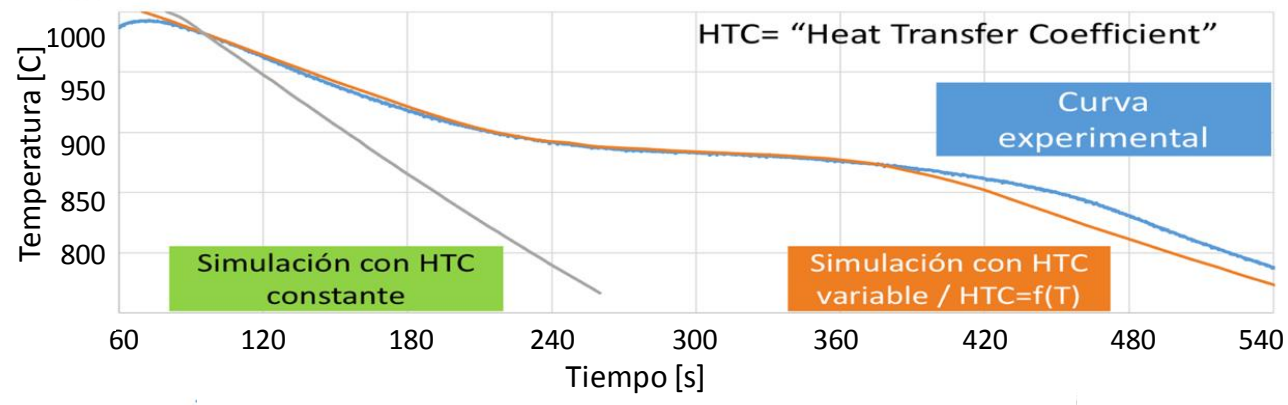

Figura 14: Ajuste de coeficiente de transmisión de calor (HTC) en Simufact.Forming para el canal 1.

Un esquema del modelo computacional se observa en la Figura 15.
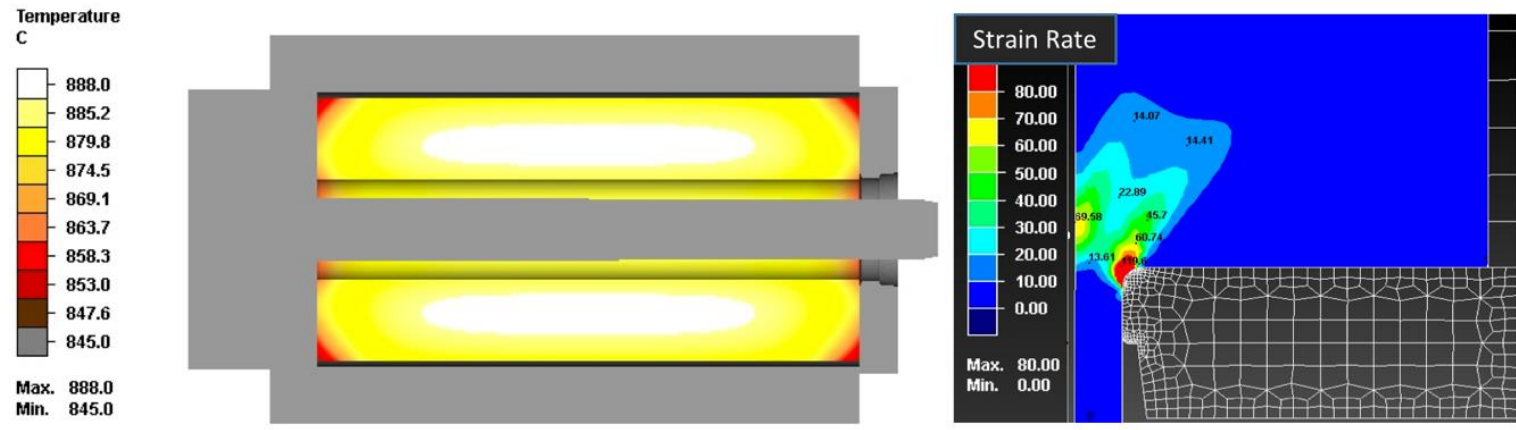

Figura 15: Modelo de elementos finitos para extrusión. Izq: temperatura inicial del billet (horizontal). Der: velocidad de deformación durante la extrusión (vertical).

Los resultados de las simulaciones se detallan en la Figura 16 junto con los resultados experimentales. Se puede observar que el modelo se ajusta satisfactoriamente a los resultados obtenidos en la planta. En 2 de los 3 casos el modelo estimó fuerzas de extrusión dentro de un 5\% de lo relevado a pie de máquina. En el caso restante el modelo subestimó la fuerza de extrusión en un $10 \%$ aproximadamente. Si bien futuras pruebas y ensayos permitirán refinar el modelo aun más, en base a los resultados detallados en [1] es posible estimar satisfactoriamente fuerzas de extrusión y evaluar el efecto de la temperatura, los tiempos muertos del proceso y la utilización de distintos lubricantes en pruebas de extrusión a escala real de productos cilíndricos y tubulares de Ti CP Gr 2. 


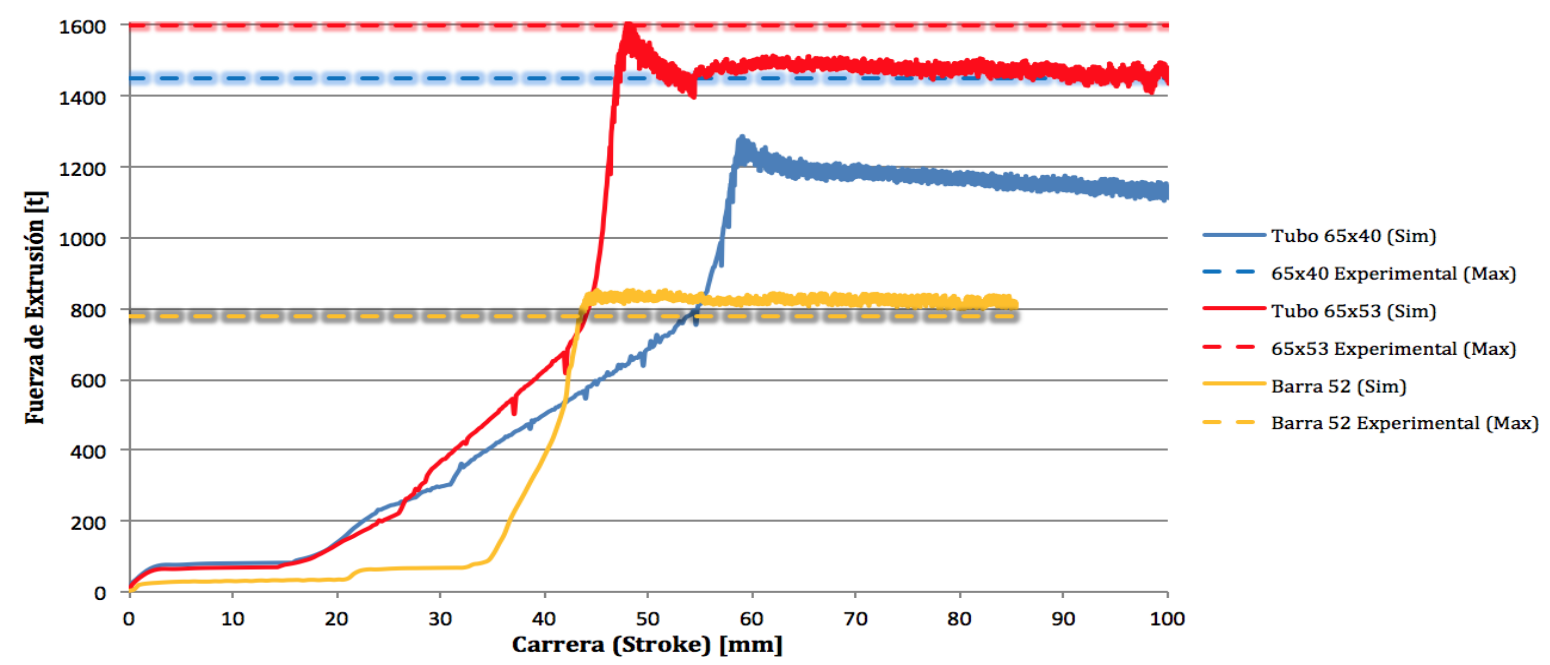

Figura 16: Fuerza de extrusión. Resultados de la simulación por elementos finitos.

\section{CONCLUSIONES}

1. El ensayo de torsión en caliente resulta un método muy efectivo para obtener información del comportamiento de los materiales, durante la deformación en caliente.

2. Los datos obtenidos del ensayo son de aplicación directa en las plantas, para definir o modificar parámetros de proceso, en aleaciones actualmente utilizadas y/o en desarrollo.

3. El amplio rango de variables de operación de la máquina para ensayos de torsión en caliente permiten simular diversas secuencias de procesamiento de una manera sencilla y rápida.

4. Desde el punto de vista microestructural, los resultados indican que las rutas de procesamiento ensayadas permiten obtener productos compatibles con las condiciones de aceptación solicitadas por las normas internacionales vigentes.

5. Las microestructuras obtenidas en las simulaciones resultaron muy finas, lo que permite ajustar los parámetros del recocido dentro de un rango amplio.

6. Las microestructuras obtenidas están comprendidas dentro de los criterios de aprobación definidos por la norma ETTC-2, para productos de calidad superior a la standard.

7. Se verificó que la cantidad de deformación necesaria en rango $\alpha+\beta$ para obtener una estructura satisfactoriamente equiaxiada en Ti-6Al-4V equivale a una reducción de área mínima de 50\%.

8. El modelado por elementos finitos del proceso de extrusión de productos cilindros y tubulares de Ti CP Gr 2 entregó resultados satisfactorios y permitió calcular la fuerza de extrusión necesaria en procesos a escala real con aceptable precisión.

\section{AGRADECIMIENTOS}

Los autores de este trabajo quisieran destacar la colaboración de las siguientes instituciones:

- A todo el personal de FAE S.A. y en particular al equipo de trabajo del sector fundición.

- A la CNEA y en particular a los integrantes del laboratorio de metalografía y microscopía del Centro Atómico Constituyentes y al Instituto Sabato.

- Al la UT: Máquinas y Herramientas del Centro INTI - Mecánica.

- Al IAS y en particular a los responsables de la máquina de torsión en caliente.

\section{BIBLIOGRAFÍA}

[1] ABATE, G., DEFERRARI, N., KRAHMER, D., et al., Failure analysis and simulation of a CP Titanium (Gr 2) hot-extrusion process using SIMUFACT.Forming, In: $36^{\circ}$ Senafor $-21^{\text {a }}$ Conferência Internacional de Forjamento, Porto Alegre, Brasil, 5-7 Octubre 2016.

[2] DONACHIE, M.J., Titanium: A Technical Guide, 2 ed, Materials Park, OH, ASM International, 2000.

[3] FROES, F.H., Titanium: Physical Metallurgy, Processing, and Applications, 1 ed, Materials Park, OH, ASM International, 2015. 
[4] LÜTJERING ,G., WILLIAMS, J.C., Titanium, 2 ed, New York, Springer, 2007.

[5] DIETER, G.E., Mechanical Metallurgy, 3 ed, New York, McGraw-Hill Education, 1986.

[6] DIETER, G.E., Handbook of Workability and Process Design, 1 ed., SEMIATIN, S.L., KUHN, H.A., editors, Materials Park, OH, ASM International, 2003.

[7] MERLONE, G.F., NUÑEZ PETTINARI, S.I., Atlas de trabajabilidad en caliente de aceros, IAS, 1993.

[8] SELLARS, C.M., TEGART, W.J.M., "Hot Workability", International Metallurgical Reviews, v. 17, n. 1, pp. 1-24, Jan. 1996.

[9] FIELDS, D.S., BACKOFEN, W.A., "Determination of Strain-Hardening Characteristics By Torsion Testing", Proceedings - ASTM, v. 57, pp. 1259-72, 1957.

[10] PRASAD, Y.V.R.K., SESHACHARYULU, T., "Processing maps for hot working of titanium alloys", Materials Science and Engineering A, v. 243, n. 1-2, pp. 82-88, Mar 1998.

[11] PRASAD, Y.V.R.K., RAO, K.P., SASIDHARA, S., Hot Working Guide: A Compendium of Processing Maps, 2 Ed, Materials Park, OH, ASM International, 2015.

[12] TECHNICAL COMMITTEE OF EUROPEAN TITANIUM PRODUCERS, ETTC 2 - Microstructural standards for alpha+beta titanium alloy bars, 2 ed, TIG - Titanium Information Group, 1995.

[13] ISO 20160, Implants for surgery - Metallic materials - Classification of microstructures for alpha+beta titanium alloy bars, ISO - International Organization for Standardization, 2006.

[14] SIENIAWSKI, J., ZIAJA, W., KUBIAK, K., MOTYKA, M., "Microstructure and Mechanical Properties of High Strength Two-Phase Titanium Alloys, Titanium Alloys - Advances in Properties Control", 1 ed, InTech, 2013. Disponible en: https://www.intechopen.com/books/titanium-alloys-advances-in-propertiescontrol/microstructure-and-mechanical-properties-of-high-strength-two-phase-titanium-alloys 